\title{
NO38 expression and nucleolar counts are correlated with cellular DNA content but not with proliferation parameters in colorectal carcinomas
}

\author{
Paula M De Angelis, Trond Stokke, Ole Petter F Clausen
}

\begin{abstract}
Aims-To investigate the expression of nucleolar protein $\mathrm{NO38}$, to determine the numbers of nucleoli per cell, and to examine the relations of these nucleolar parameters to tumour DNA index, total cellular DNA content, $S$ phase fraction, and Ki67 labelling index.

Methods-36 colorectal tumours and 14 normal mucosas were studied. An antiNO38 monoclonal antibody, 31A12, and flow cytometric analysis were used to detect expression of NO38 by means of a biotin-streptavidin-FITC (fluorescein isothiocyanate) staining method. Nucleolar counts were determined using fluorescence microscopy. Flow cytometry was used to determine tumour DNA indices and the sizes of the $\mathbf{S}$ phase fractions. Ki67 labelling indices were determined from tissue sections stained immunohistochemically with the MIB-1 antibody against the Ki67 nuclear protein.
\end{abstract}

Results-Generally, tumour cell nucleoli were larger and more irregular in shape compared with nucleoli in normal mucosal cells. DNA aneuploid and diploid tumours expressed 2.8 and 2.1 times more NO38 than normal mucosa. The mean (SD) values for nucleolar counts were higher for the DNA aneuploid tumours (3.81 (0.93)) than the diploid tumours (2.62 (0.38)) and normal mucosa (2.34 (0.37)). NO38 expression and numbers of nucleoli correlated significantly $(r=0.52$, $\mathbf{p}=\mathbf{0 . 0 1}$ ). There were, however, no significant correlations between these nucleolar parameters and either the sizes of tumour $S$ phase fractions or Ki67 labelling indices. Cell cycle resolved expression of NO38 in tumours and normal mucosa demonstrated that expression increased approximately in proportion to the DNA content throughout the cell cycle. In aneuploid tumours, NO38 expression was $43 \%$ and $98 \%$ higher in $S$ and $G_{2}$ phases, respectively, compared with the $G_{1}$ phase. Sorting of these populations revealed that the nucleolar count also increased as the DNA content increased but by only $29 \%$ and $47 \%$ in $S$ and $G_{2}$, respectively. Apoptotic cells lacked NO38.

Conclusions-NO38 expression is higher in tumours than in normal mucosa owing to the increased DNA content and larger nucleoli in tumours; expression increases proportionally with DNA content as cells progress through the cell cycle from $G_{1}$ through $S$ and $G_{2}$. However, NO38 expression does not correlate with the tumour $S$ phase fraction or Ki67 labelling index and is lost during apoptosis. Also the results suggest that nucleoli grow in size during the cell cycle, which would account for the doubling of NO38 expression from $G_{1}$ to $G_{2}$, as the nucleolar count increased by only $47 \%$.

(F Clin Pathol: Mol Pathol 1997;50:201-208)

Keywords: NO38 expression; nucleolar counts; DNA index; cellular DNA content; $S$ phase fraction; Ki67 labelling index; colorectal tumours

NO38, also known as B23, nucleophosmin, or numatrin is an abundant nucleolar phosphoprotein of $38 \mathrm{kDa}$ molecular weight, which is localised to the granular regions of the nucleolus, ${ }^{1}$ where maturation, processing, and storage of preribosomal particles have been shown to take place. ${ }^{23}$ Recent studies have shown NO38 to have DNA binding activity ${ }^{5}$ and to bind to another nucleolar protein, namely p120. ${ }^{6} \mathrm{NO} 38$ has been shown also to be a nuclear substrate of protein kinase $\mathrm{C}^{7}$ mitotic cdc2 kinase, ${ }^{8}$ and casein kinase II. ${ }^{9}$ The functional significance of these activities of NO38 is unknown, although it is clear that NO38 plays some role in cellular proliferative activity. This is suggested also by another study demonstrating that NO38 expression increases rapidly during mitogenic stimulation. ${ }^{10}$ In addition, NO38 may play a role in the apoptotic pathway, as suggested by a recent study $^{11}$ which demonstrated reduced NO38 expression in apoptotic Jurkat T lymphoblasts.

NO38 is one of several proteins known to be associated with nucleolar organiser regions (NORs) and to be stained by the argyrophilic nucleolar organiser region (AgNOR) technique ${ }^{12}{ }^{13}$; only NORs that are transcriptionally active are assumed to be identified by this technique..$^{14}$ Quantitative measurement of the numbers of NORs in a cell is one method used currently to assess cellular proliferative activity in human tumours. If large numbers of NORs within malignant cells reflect increased DNA synthesis (relative to benign tissue) then it may be reasonable to expect that AgNOR counts could be correlated with other cell proliferative markers such as the $\mathrm{S}$ phase fraction, BrdU labelling index, Ki67 labelling index, and others. Tumour ploidy status and S phase fraction can be determined from DNA content 


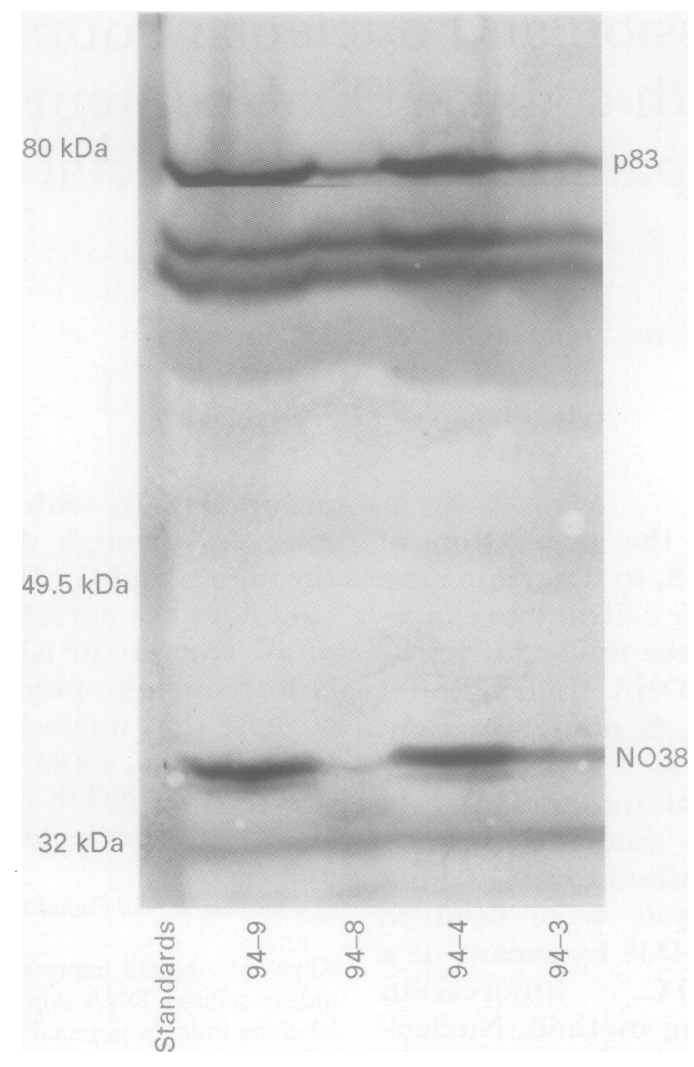

Figure 1 A répresentative immunoblot of colorectal tumours stained for NO38 and p83. A major band at $38 \mathrm{kDa}$ was seen for all tumours and a minor band directly below the major band in two tumours (94-4 and 94-9), representing different phosphorylation states of NO38. p83 was used as a concentration and loading standard.

analysis of tumour specimens using flow cytometry. The DNA index is used to report the degree of abnormal DNA content in a tumour. Both the DNA index and $S$ phase fraction have been used as prognostic indicators in colorectal cancer; the DNA index has had only modest impact in this regard. ${ }^{15}$ Generally, other parameters such as Dukes' stage and histological grade have been shown to be better prognostic factors. However, a high $S$ phase fraction has been shown to correlate with DNA aneuploidy ${ }^{16}$ and, in another study, the $S$ phase fraction proved to be a stronger predictor of survival than DNA ploidy status. ${ }^{17}$ Ideally, these factors should be reported along with stage and grade. The nuclear protein which is labelled by the Ki67 and MIB-1 antibodies is detected in proliferating but not quiescent cells. ${ }^{18} 19$ Thus, nuclei reacting with these antibodies are considered to represent the cellular growth fraction both in normal tissues and tumours.

In this study we examined the relations between both NO38 expression and the numbers of nucleoli and the tumour DNA index, total cellular DNA content, $S$ phase fraction, and Ki67 labelling index in colorectal tumours and normal mucosa. NO38 expression and nucleolar counts were higher in the tumours than in normal mucosa and highest in the DNA aneuploid tumours. In cycling aneuploid tumour cells, NO38 expression doubled while nucleolar counts increased by only $47 \%$ as the cells progressed from $G_{1}$ to $G_{2}$, implying that the nucleoli grow in size during the cell cycle. Neither NO38 expression nor the numbers of nucleoli correlated significantly with the tumour S phase fraction or Ki67 labelling index.

\section{Materials and methods}

CLINICAL MATERIAL

Thirty six surgically removed and previously untreated colorectal carcinomas and 14 normal colonic mucosal specimens were collected and studied. All tumours were classified according to their Dukes' stage ${ }^{20}$ : one was Dukes' A, 18 were Dukes' B, 10 were Dukes' C, and seven were Dukes' D. Normal mucosal samples were selected from macroscopically normal areas of surgical specimens. Samples of fresh tumour and normal mucosa were frozen without buffer at $-80^{\circ} \mathrm{C}$ immediately following surgery.

DETECTION OF NO38 PROTEIN BY

IMMUNOBLOTTING

Slices of tumour and normal tissue (0.5 $1.0 \mathrm{~mm}$ ) were boiled for three to four minutes in sodium dodecyl sulphate (SDS) sample buffer. ${ }^{21}$ The NO38 protein and prestained broad range molecular weight standards (BioRad, California, USA) were separated by electrophoresis in $10 \%$ SDS polyacrylamide gels. ${ }^{21}$ Immunoblotting was performed as described previously, ${ }^{22}$ using the mouse antihuman NO38 monoclonal antibody 31A12 (T Stokke, unpublished) and a biotin-streptavidin alkaline phosphatase staining procedure to detect 31A12 (Amersham, Amersham, UK). p83 is a protein distributed abundantly throughout the nucleoplasm of all human cells tested. The amount of $\mathrm{p} 83$ per cell does not appear to vary with growth rate or tissue origin ${ }^{22}{ }^{23}$ and was determined on the same blots as a control for gel loading and cell concentration.

IMMUNOFLUORESCENCE STAINING OF NO38 AND DETERMINATION OF THE MEAN NUMBERS OF NUCLEOLI PER CELL BY FLUORESCENCE

MICROSCOPY

Single nuclear suspensions were prepared from tumour and normal mucosal material by mincing tissue slices in $10 \mathrm{mM}$ phosphate buffer $N$ containing $0.1 \%$ Triton $\mathrm{X} 100,5 \mathrm{mM} \mathrm{MgCl}$, $10 \mathrm{mM} \mathrm{NaCl}$, and $0.1 \mathrm{mM}$ phenylmethylsulphonyl fluoride (PMSF). Each tumour sample contained a mixture of cells from at least three separate areas of the tumour specimen. The suspensions were placed on ice $\stackrel{\mathbb{D}}{?}$ for 10 minutes, filtered, fixed for one hour on ice by the addition of $1 \%$ paraformaldehyde, 0 washed once with phosphate buffered saline (PBS), centrifuged, resuspended in PBS con- $\mathbb{\otimes}$ taining $0.1 \%$ bovine serum albumin (BSA), and aliquoted as $100 \mu \mathrm{l}$ suspensions $\left(1-2 \times 10^{6}\right.$ cells). PBS containing $0.1 \%$ Triton X100 was added for five minutes on ice followed by addition of dry milk (added to a final concentration of $5 \%$ ). The suspension was incubated for 15 minutes at room temperature, after which the anti-NO38 monoclonal antibody $31 \mathrm{~A} 12$ was added at a 1/50 dilution of the stock supernatant and incubated for one hour on ice. Control samples received no primary antibody. Samples were then washed once with PBS $/ 0.1 \%$ 


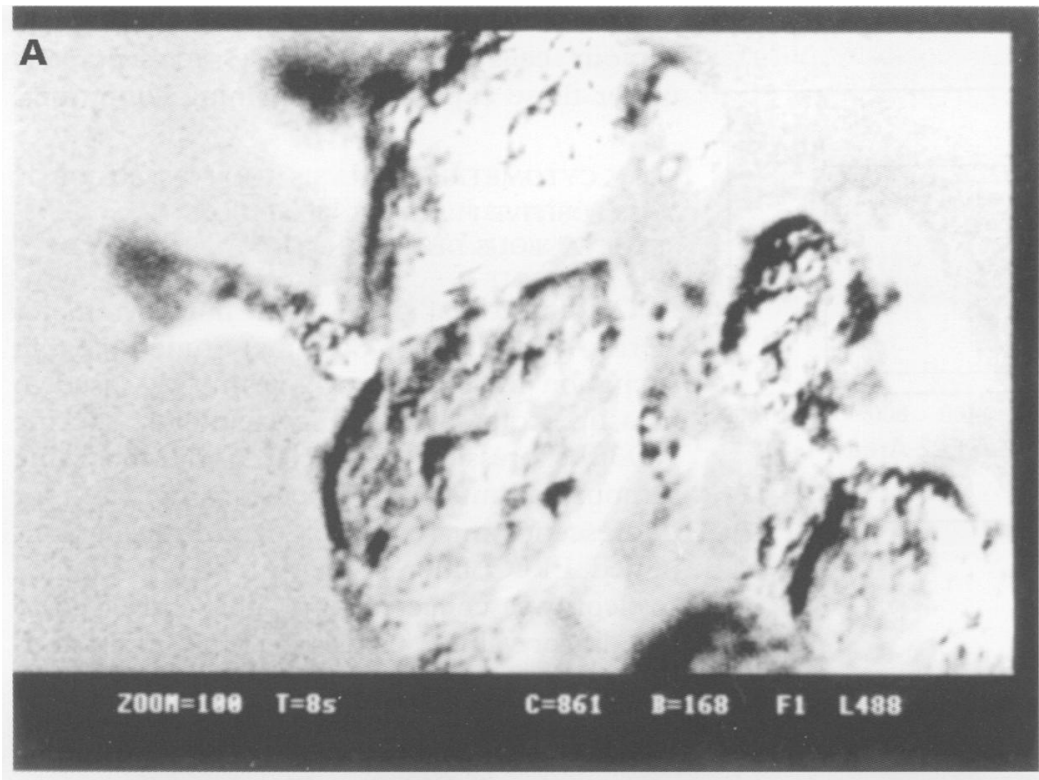

B
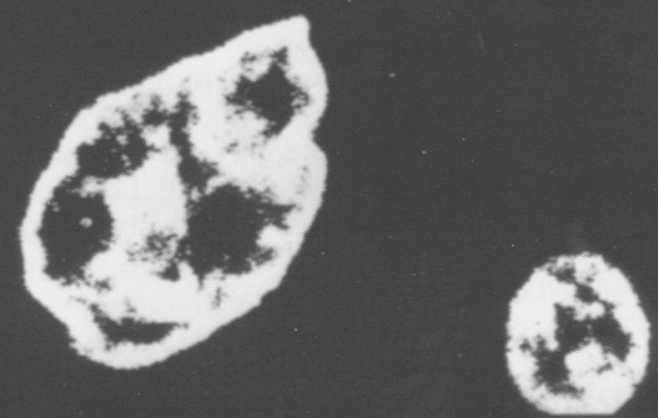

$200 \mathrm{H}=108 \quad \mathrm{~T}=8 \mathrm{~s}$

$C=516 \quad B=8$

F1 1514

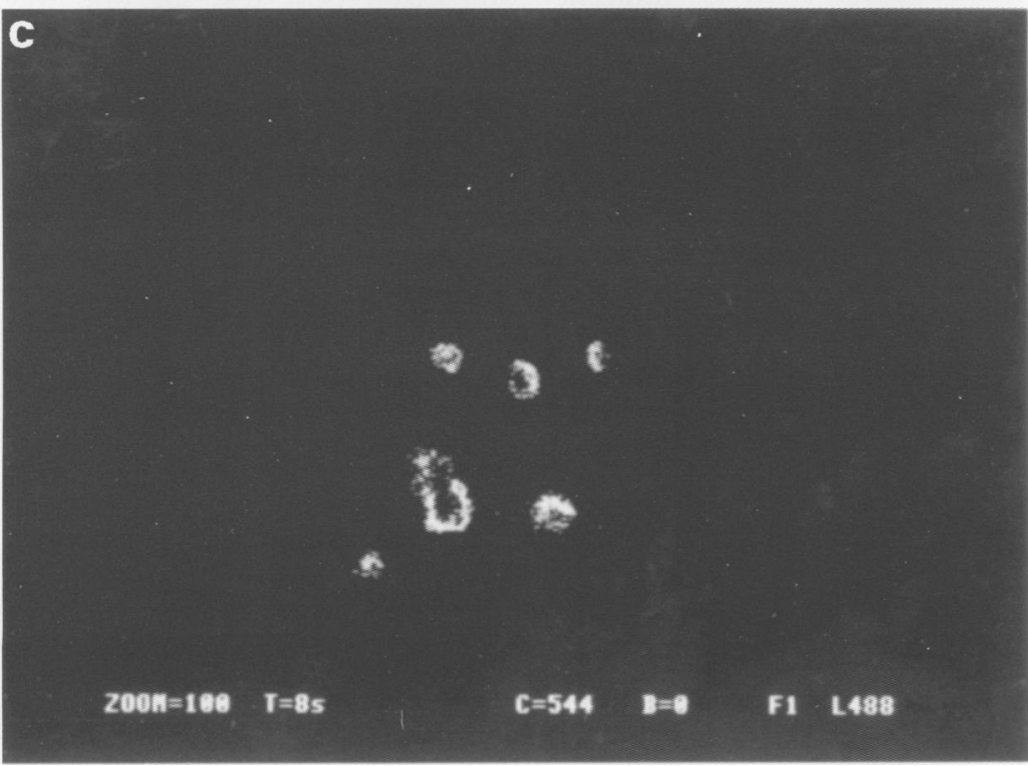

Figure 2 Laser confocal micrographs of colorectal tumour nuclei stained with the anti-NO38 antibody $31 A 12$ and counterstained with propidium iodide for total DNA. (A) Phase contrast micrograph of the nuclei of interest; $(B)$ propidium iodide fluorescence of the same nuclei showing the distribution of total DNA in the nucleus; (C) FITC fluorescence of the same nuclei stained for NO38.
Triton X100, followed by addition of the secondary antibody (biotinylated horse antimouse IgG (Vector Labs, California, USA), made up as a $1 / 35$ dilution in PBS plus 5\% dry milk) for 20 minutes. The washing step was repeated, followed by addition of the tertiary reagent, streptavidin-FITC (Amersham) made up as a $1 / 35$ dilution in PBS plus $5 \%$ dry milk for 20 minutes. The samples were washed with PBS/0.1\% Triton X100 and the pellets resuspended in PBS with propidium iodide (PI) $(5 \mu \mathrm{g} / \mathrm{ml})$. Aliquots of these suspensions were examined using a Nikon Labophot fluorescence microscope (Nikon Corp, Tokyo, Japan) at a $40 \times$ magnification, with an FITC emission filter setup. The nucleoli were counted in a field of 100 cells per tumour or normal mucosal sample and the mean values calculated.

LOCALISATION OF NO38 PROTEIN BY LASER

CONFOCAL MICROSCOPY

Several of the same tumour suspensions used for fluorescence microscopy were examined also by laser confocal microscopy (Zeiss, Oberkochen, Germany). FITC emission (510$550 \mathrm{~nm}, 488 \mathrm{~nm}$ excitation) was measured to determine the localisation of the NO38 protein in the nucleolus, whereas total DNA was visualised with propidium iodide (PI) (emission $>620 \mathrm{~nm}, 514 \mathrm{~nm}$ excitation). Non-confocal interference contrast images $(488 \mathrm{~nm})$ were also generated for the same cells.

IMMUNOHISTOCHEMICAL DETECTION OF THE KI67 PROTEIN AND DETERMINATION OF THE KI67

LABELLING INDEX

Formalin fixed, paraffin wax embedded tissue sections $(3 \mu \mathrm{m})$ were deparaffinised, dehydrated in ethanol, and rinsed in distilled water. For antigen unmasking, the slides were microwaved at $750 \mathrm{~W}$ three times for five minutes each, in $10 \mathrm{mM}$ citrate buffer ( $\mathrm{pH} \mathrm{6.0)}$. The slides were cooled for 30 minutes at room temperature, followed by washing in Tris buffer (pH 7.4) for five minutes. They were then incubated with the MIB-1 mouse antihuman Ki67 monoclonal antibody (1/50 dilution of stock antibody which was a gift from Dr J Gerdes, Germany) overnight at $4^{\circ} \mathrm{C}$. Positive and negative controls (without the primary antibody) were included in the staining protocol. The sections were then washed with Tris buffer and the bound antibody visualised using the conventional alkaline phosphatase antialkaline phosphatase (APAAP) method. Evaluation of the sections and scoring for positivity were carried out by one of us (OPFC). Tumour cells with evident nuclear staining were scored as positive and 300-500 cells were counted randomly. Preliminary counts showed that some cells $(<10 \%)$ had relatively weak nuclear staining, thus all positive cells were pooled and reported as one count. The percentage of positive cells was calculated for each tumour and reported as the Ki67 labelling index. 
A

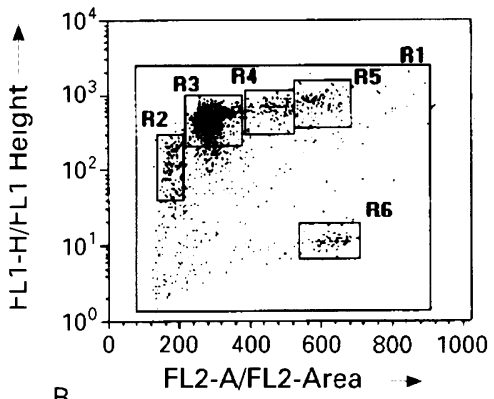

B

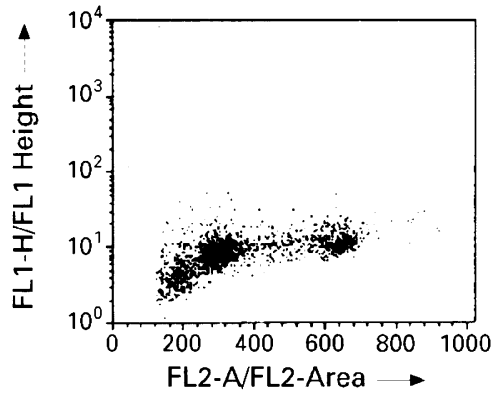

C

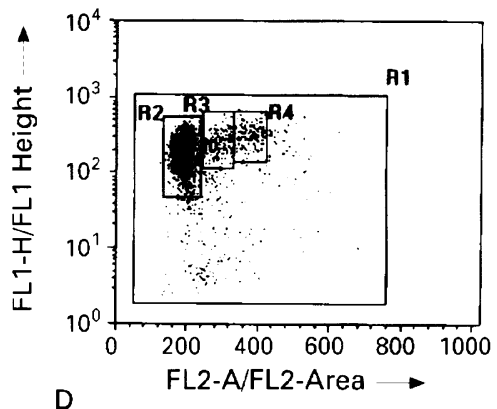

D

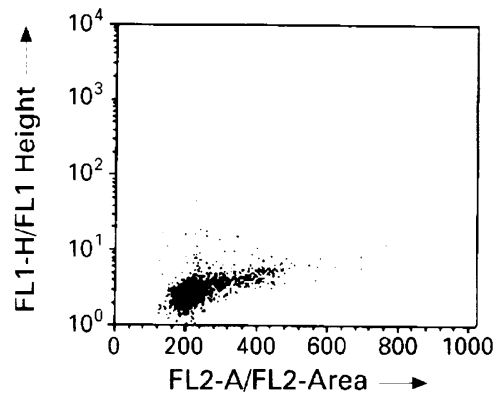

Figure 3 (A) Tumour 94-12 stained with the anti-NO38 antibody 31 A12 (FL1) and propidium iodide (PI) for DNA (FL2). The regions $R 2, R 3, R 4$, and $R 5$ were generated for analysis and sorting purposes, and represent the diploid $G_{i}$, aneuploid $G_{i}$, aneuploid $S$, and aneuploid $G$.components in this aneuploid tumour, respectively; $R 6$ represents cells that have high PI fluorescence and were shown to be apoptotic. (B) The same tumour, stained as under $(A)$ but without the primary antibody $31 A 12$ (fluorescence control). (C) Tumour 94-22 stained as under $(A)$ with $31 A 12$ primary antibody; $R 2, R 3, R 4$ represent the diploid $G_{i}, S$, and $G_{2}$ components, respectively. (D) Fluorescence control for the same tumour. reason the $S$ phase fraction of the diploid tumour cells cannot be assessed separately under these experimental staining conditions.

FLOW CYTOMETRIC ANALYSIS AND SORTING OF NO38 POSITIVE FRACTIONS BASED ON

SIMULTANEOUS DETERMINATION OF NO38 AND DNA CONTENT

A Cytofluorograf $50 \mathrm{H}$ laser flow cytometer (Ortho Diagnostic Systems) tuned to an excitation wavelength of $488 \mathrm{~nm}$ was used to measure total and cell cycle/ploidy specific NO38 expression in nuclei using the same tumour and mucosal suspensions prepared for fluorescence microscopy. Logarithmically amplified FITC fluorescence of NO38 labelled nucleoli was collected (and later converted to true linear values) using a 515-530 nm bandpass filter, and PI fluorescence was collected as described above. Fluorescence values for í NO38 expression are given as arbitrary fluorescence units. A FACStar Plus laser flow cyto- ĩ meter (Becton-Dickinson, California, USA) tuned to an excitation wavelength of $488 \mathrm{~nm}$ 옹 was used to sort various NO38 positive fractions (for example, $G_{1}, S$, and $G_{2}$ phase cells) in 10 tumours (aneuploid and diploid) and three normal mucosa based on bivariate distributions of green fluorescence (log scale FITC fluorescence of NO38 stained nucleoli) versus red fluorescence (DNA content of PI stained nuclei). The emission filter setups were essentially the same as for the Ortho Cytofluorograf. The mean numbers of nucleoli per cell in each sorted fraction were calculated based on 100 cells sorted/examined.

\section{STATISTICAL ANALYSIS}

Unpaired non-parametric tests (MannWhitney, two tailed) were used to check for significant differences between two groups of data for a specific parameter. The Bonferroni multiple comparison post test for one way analysis of variance (ANOVA) was used if the same parameter was compared in three or 음 more groups. Fisher's two-tailed $2 \times 2$ contin- $\frac{7}{0}$ gency tests were used to check for associations 을 between any two parameters, and correlation $N$ analysis or linear regression analysis were used $N$ to check for the degree of covariation between $\mathrm{N}$ two variables. All statistical testing was performed using Prism software (Graphpad Software, California, USA). $\mathrm{p}$ values $<0.05$ were considered to be significant.

\section{Results}

SUBNUCLEOLAR LOCALISATION OF NO38 AND NUCLEOLAR PLEOMORPHISM

Figure 1 shows a representative immunoblot of colonic tumours stained with the $31 \mathrm{~A} 12$ antibody. The protein recognised by this antibody banded at $\sim 38 \mathrm{kDa}$ (major band); a minor band could be observed directly under- 륵 neath the major band in two tumours (94-4 and 94-9).

Both conventional fluorescence microscopy and laser confocal microscopy demonstrated that the $31 \mathrm{~A} 12$ antibody stained nucleoli specifically (fig 2). Thus, data from immunoblotting as well as from microscopy was 

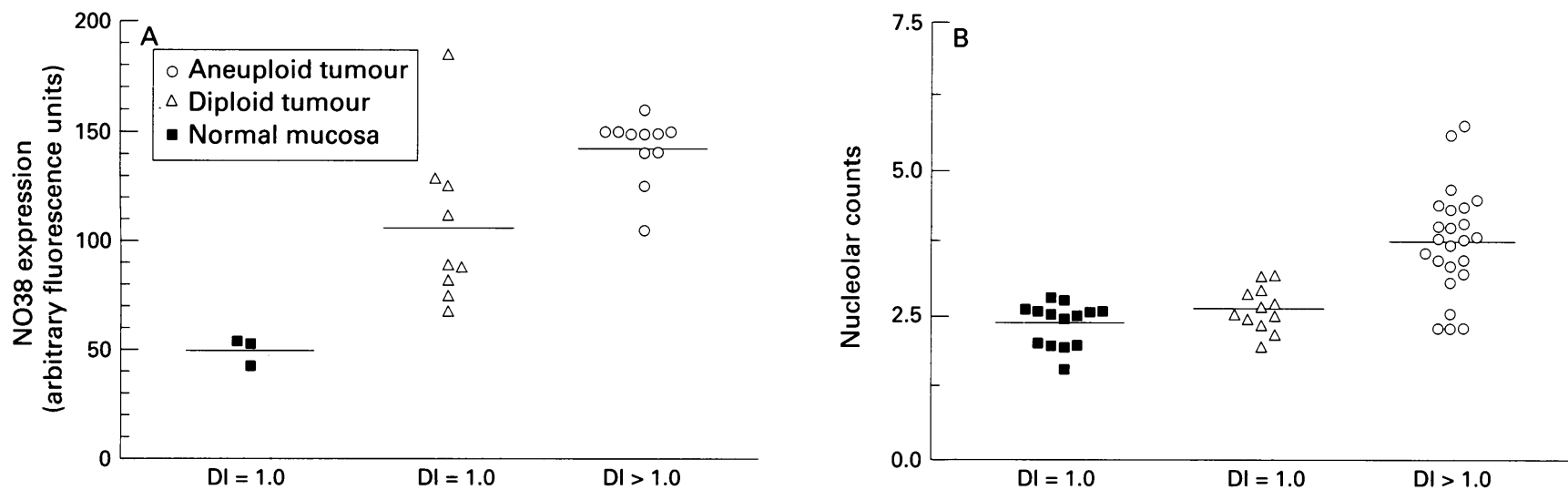

Figure 4 (A) NO38 expression of the total ungated positive cell population (mean FITC fluorescence of NO38 labelled cells measured in arbitrary fluorescence units) is highest in DNA aneuploid colorectal tumours compared with diploid tumours and normal mucosa. (B) Numbers of nucleoli (mean number of nucleoli per cell) are also highest in DNA aneuploid tumours relative to diploid tumours and normal mucosa. The horizontal lines represent the mean value for each dataset.

consistent with the observation that $31 \mathrm{~A} 12$ recognises the nucleolar protein NO38. The minor bands seen in fig 1 probably represented different phosphorylation states of the protein. NO38 immunofluorescence appeared to be localised to the perimeter of the nucleoli, such that the nucleolar centre appeared black, surrounded by a ring of positive staining. The tumour nuclear suspensions were a mixture of tumour and normal mucosal nuclei, and the number of nucleoli seen/counted per nucleus ranged from one to 16 for the entire tumour set. All specimens contained a certain number of nuclei that did not demonstrate apparent nucleolar staining (non-fluorescent). In the tumours, there was a distinct heterogeneity in nucleolar sizes, ranging from small to very large, and in shape, with irregular patterns including round and elongated nucleoli. The nucleoli seen/counted were distinct bodies and not fluorescent "specks". In the normal mucosal specimens the numbers of nucleoli per nucleus ranged from one to six, and they appeared generally to be smaller and more rounded than those seen in the tumour specimens-that is, more homogeneous with respect to size and shape.

NO38 EXPRESSION AND NUCLEOLAR COUNTS

Flow cytometric analysis demonstrated that all tumours and normal mucosal specimens expressed NO38, but at variable levels of intensity (examples are shown in fig 3). NO38 expression was not restricted to one cell cycle compartment but was expressed throughout the cycle. The diploid, presumably normal, cells in aneuploid tumours also expressed the protein (fig 3A, region R2). A few aneuploid tumours contained a small population of NO38 negative cells with high PI staining (fig $3 \mathrm{~A}$, region $\mathrm{R} 6$ ). These were sorted using flow cytometry and examined microscopically; most of these cells had the morphological characteristics of apoptotic cells.

Total NO38 expression for each sample was obtained from the flow cytometric data as the mean FITC intensity of the whole, ungated positive population; the mean (SD) values for total NO38 expression were 142 (15) for the aneuploid tumours, 106 (37) for the diploid tumours, and 50 (6) for normal mucosa (fig
4A). Nucleolar counts were obtained from fluorescence microscopy data and mean values for this parameter were $3.81(0.93)$ for the aneuploid tumours, $2.62(0.38)$ for the diploid tumours, and $2.34(0.37)$ for normal mucosa (fig 4B). NO38 expression correlated significantly with the numbers of nucleoli $(r=0.52$, $\mathrm{p}=0.01)$. NO38 expression and nucleolar counts were significantly higher in the tumours than in normal mucosa $(p=0.009$ and $p=0.001$, respectively), and highest in the DNA aneuploid tumour group compared with the diploid tumour group $(\mathrm{p}<0.05$ and $\mathrm{p}<0.001$, respectively).

No significant differences were observed for either NO38 expression or nucleolar counts between Dukes' stage C $+D$ and Dukes' $A+B$ tumours ( $p=0.13$ and $p=0.43$, respectively).

KI67 LABELLING INDEX AND S PHASE FRACTION The mean (SD) Ki67 labelling indices were 70 (18) for the aneuploid tumours, 72 (19) for the diploid tumours, and 38 (4) for the normal mucosal specimens. The mean percentages of $S$ phase cells were 12.3 (7.0) for the aneuploid tumours, 15.9 (4.2) for the diploid tumours, and 7.0 (2.8) for the normal mucosal specimens. There was no significant correlation between the tumour $S$ phase fraction and the tumour Ki67 labelling index $(r=0.17$, $\mathrm{p}=0.40$ ). In the tumour group, there were no significant correlations between NO38 expression and either the size of the $\mathrm{S}$ phase fraction or the Ki67 labelling index $(r=0.17$ and $\mathrm{p}=0.46 ; r=0.15$ and $\mathrm{p}=0.65$, respectively); the same was true for nucleolar counts versus these two parameters $(r=0.29$ and $\mathrm{p}=0.09$; $r=0.10$ and $\mathrm{p}=0.62$, respectively).

NO38 EXPRESSION, NUCLEOLAR COUNTS, AND DNA CONTENT

It is possible that the higher NO38 expression and nucleolar counts seen in the tumour group compared with normal mucosa could be a result of the higher DNA indices in the tumour group. Therefore, NO38 expression and the numbers of nucleoli were compared in the diploid tumours and normal mucosa. NO38 expression was significantly higher in the former than in the latter $(p<0.05)$, whereas 

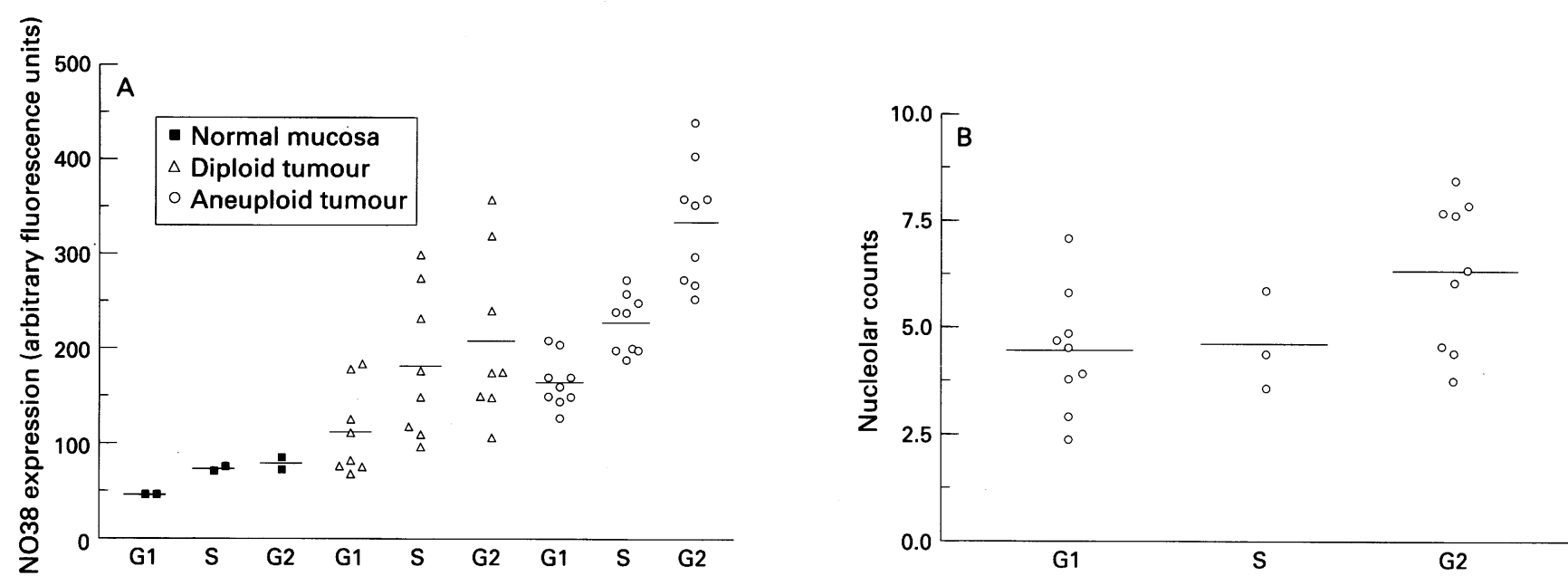

Figure 5 (A) NO38 expression in tumours and normal mucosa increases as cells progress from $G_{1}$ through $S$ to the $G_{2}$ phase. (B) Nucleolar counts increase as aneuploid tumour cells progress through the cell cycle. Horizontal lines represent the mean value for each dataset.

the numbers of nucleoli were not. If NO38 expression and nucleolar counts in the aneuploid tumours were divided by the DNA index and compared with normal mucosa, the same trend was seen. This implies that tumour cells express higher amounts of NO38 in relation to the amount of DNA compared with normal mucosal cells, but that they contain the same numbers of nucleoli relative to the amount of DNA as do normal mucosal cells.

It was directly evident from the flow cytometric cytograms of NO38 fluorescence versus DNA content that NO38 expression increased as the DNA content increased throughout the cell cycle of the aneuploid tumour populations (fig 3A). In diploid tumours, diploid cells (fig 3A, R2 and fig 3C, R2) showed a more heterogeneous expression of NO38 than aneuploid cells and the cells in the $S$ and $G_{2}$ phases of the cell cycle. This might be because in diploid tumours, diploid cells consist of leucocytes and normal mucosal cells in addition to tumour cells. Thus, aneuploid tumour cells represent the most homogeneous population, a fact that can be distinguished clearly by DNA content. However, $S$ and $G_{2}$ cells from diploid tumours are also relatively pure because the admixture of non-tumour cells comprises mainly nonproliferating leucocytes and mucosal cells. Therefore, the cell cycle resolved expression of NO38 in a presumably homogeneous population of tumour cells was analysed in more detail in the aneuploid tumours. NO38 (SD) expression was $43 \%(29 \%)$ and $98 \%(48 \%)$ higher in the $S$ and $G_{2}$ phases, respectively, relative to its expression in $G_{1}$ (fig $5 \mathrm{~A}$ ). In addition, these aneuploid tumours were sorted using flow cytometry to determine the number of nucleoli in the $S$ and $G_{2}$ phases relative to $G_{1}$. The numbers (SD) of nucleoli were $29 \%$ (18\%) higher in $S$ than in $G_{1}$, and the numbers of nucleoli were $47 \%(23 \%)$ higher in $\mathrm{G}_{2}$ than in $\mathrm{G}_{1}$ (fig 5B). Hence, on average, the nucleoli must grow in size to contain $35 \%$ more NO38 as the cells proceed from $G_{1}$ to $G_{2}$.

\section{Discussion}

We examined the relation between both NO38 expression and nucleolar counts and the DNA index, total cellular DNA content, $S$ phase fraction, and Ki67 labelling index in colorectal i carcinomas, using a monoclonal antibody that specifically labels NO38. This is a nucleolar 을 phosphoprotein associated with nucleolar organiser regions in cells. The immunofluorescent staining of NO38 was localised specifically to the perimeter of the nucleoli, which is $\stackrel{5}{\rightarrow}$ consistent with a previous report demonstrat- $\vec{\theta}$ ing NO38 localisation to the outer granular regions (granular and dense fibrillar components) of the nucleoli. ${ }^{1}$ The pattern of staining in tumours was clearly different from that seen in normal mucosa. In general, tumour nucleoli were larger and more irregular in shape (round, $\mathbb{D}$ oval, or elongated) compared with nucleoli seen in normal mucosa. Nucleolar pleomorphism has been known for some years to be characteristic of cancer cells. ${ }^{26}$ The tumour group also had significantly higher nucleolar counts than the normal mucosa group. It has been shown previously with AgNOR staining that, generally, highly malignant neoplasms have smaller and more numerous AgNORs than benign or less malignant tumours. ${ }^{28} 29$ Yang et $a l^{30}$ demonstrated a higher number of AgNOR counts in adenocarcinomas than in adenomas with severe dysplasia. The reasons for these differences are still unclear. Although we did not use the more conventional AgNOR technique to label nucleolar proteins, our study is consistent with the AgNOR studies as we demonstrated increased numbers of nucleoli in tumour cells compared to normal cells, although they were not smaller in the tumour group. The relation between NO38 staining and AgNOR counts is not quite resolved, and is a subject of great interest that deserves further attention.

NO38 expression was seen in all phases of the cell cycle of tumour and normal mucosal cells, and morphologically apoptotic tumour cells lacked NO38, as shown by cell sorting. Nucleolar counts correlated significantly with NO38 expression, and both parameters were significantly higher in the tumours than normal mucosa, being highest in the DNA aneuploid tumours.

No significant association was observed between nucleolar counts and Dukes' stage, in 
agreement with the results of Kram et al. ${ }^{31}$ In addition, we did not observe significant correlations between either NO38 expression or nucleolar counts and $\mathrm{S}$ or $\mathrm{S}+\mathrm{G}_{2}$ phase fractions in the tumour cell populations. These results are consistent with previous reports failing to demonstrate significant correlations between AgNOR counts and the percentage of cells in the $\mathrm{S}+\mathrm{G}_{2}$ phases of the cell cycle in colonic adenocarcinoma, ${ }^{31}$ gastric carcinoma, ${ }^{32}$ and breast cancer. ${ }^{33}$ However, Hasegawa et al $^{34}$ reported a significant linear correlation between the AgNOR count and percentage of cells in the $S+G_{2}$ phase in testicular germ cell carcinomas.

It was speculated that the observed differences in NO38 expression and nucleolar counts between the tumours and normal mucosa could be a result of the higher average DNA index in the tumour group. Both NO38 expression and the number of nucleoli increase with DNA content, but only NO38 expression appears to be higher in tumours compared with normal mucosa after normalising for DNA content. That is, per amount of DNA, both tumour cells and normal mucosal cells contain the same numbers of nucleoli. Thus, the higher NO38 expression seen in the tumours is most likely related to the larger sizes of the tumour nucleoli.

It was clear from the NO38 flow cytometric sorting analysis of aneuploid tumour cells that while NO38 expression increases by $-100 \%$ from $G_{1}$ to $G_{2}$, the nucleolar count increases by only $47 \%$. Thus, the nucleoli must increase in size to account for the observed increase in NO38 expression from $G_{1}$ to $G_{2}$. NO38 expression increases by only $85 \%$ and $70 \%$ from $G_{1}$ to $G_{2}$ in diploid tumours and normal mucosa, respectively. This is probably because of the admixture of mainly non-cycling leucocytes in the diploid component of these groups, which results in a heterogeneity of NO38 expression. Unlike the aneuploid tumours, it was not possible to examine homogeneous populations of cycling cells in these two groups. However, future studies with threeparameter NO38, keratin, and DNA flow cytometry might be possible in order to distinguish leucocytes from epithelial cells for further analysis of NO38 expression and nucleolar frequency.

Neither NO38 expression nor nucleolar counts correlated with the sizes of the tumour $\mathrm{S}$ or $\mathrm{S}+\mathrm{G}_{2}$ phase fractions, or with the $\mathrm{Ki} 67$ labelling index in colorectal carcinomas. Furthermore, no significant correlation was found between the tumour $S$ phase fraction and the Ki67 labelling index. Thus, even though NO38 expression and nucleolar counts are increased in $S$ phase and $G_{2}$ phase cells relative to $G_{1}$ phase cells, the sizes of the $S$ phase (and $S+G_{2}$ phase) fractions are relatively small compared to the sizes of the $G_{1}$ fractions. This may explain the observed lack of correlation between these nucleolar parameters and the $S$ and $S+G_{2}$ phase fractions. Thus our results indicate that NO38 expression does not correlate with cell proliferation as measured by the sizes of the $\mathrm{S}$ phase fraction or by the $\mathrm{Ki} 67$ labelling index. There was also no correlation between the $S$ phase fraction and the Ki67 labelling index, indicating that these are independent parameters, both of which may reflect cell proliferation.

NO38 expression and nucleolar counts increase with DNA content, whether this is caused by an aneuploid tumour stemline or passage through the cell cycle. The number of nucleoli is not a marker of neoplasia per se, since the increased nucleolar counts seen in tumours are the result of their increased DNA index relative to normal mucosa. NO38 expression, however, is higher in colorectal tumours than in normal mucosa, and this remains true even when the DNA content is taken into consideration. This is consistent with the observed larger sizes of nucleoli in tumour cells compared with normal mucosal cells. This work was supported by the Norwegian Cancer Society. Akershus Hospital, Oslo, Norway, for providing colorectal tumour and mucosal specimens.

1 Spector DL, Ochs RL, Busch H. Silver staining, immunofluorescence, and immunoelectron microscope localization of nucleolar phosphoproteins B23 and C23. Chromosoma 1984;90:139-48.

2 Goessens G. Nucleolar structure. Int Rev Cytol 1984;87: 107-58.

3 Jordan EG. Interpreting nucleolar structure: where are the transcribing genes? F Cell Sci 1991;98:437-42.

4 Dumbar TS, Gentry GA, Olson MOJ. Interaction of nucleolar phosphoprotein B23 with nucleic acids. Biochemistry 1989;28:9495-501.

5 Feuerstein N, Mond JJ, Kinchington PR, Hickey R, Lindsberg M-LK, Hay I, et al. Evidence for DNA binding activity of numatrin (B23), a cell cycle-regulated nuclear matrix protein. Biochim Biophys Acta 1990;1087:127-36.

6 Valdez BC, Perlaky L, Henning D, Saijo Y, Chan P-K, Busch $\mathrm{H}$. Identification of the nuclear and nucleolar localization signals of the protein p120. Interaction with translocation protein B23. F Biol Chem 1994;269:23776-83.

7 Beckmann R, Buchner K, Jungblut PR, Eckerskorn C, Weise C, Hilbert $R$, et al. Nuclear substrates of protein kinase C. Eur 7 Biochem 1992;210:45-51.

8 Feuerstein N. Phosphorylation of numatrin and other nuclear proteins by cdc2 containing CTD kinase cdc $2 / \mathrm{p} 58$. f Biol Chem 1991;266:16200-6.

9 Pfaff M, Anderer FA. Casein kinase II accumulation in the nucleolus and its role in nucleolar phosphorylation. Biochim Biophys Acta 1988;969:100-9.

10 Feuerstein N, Mond JJ. "Numatrin", a nuclear matrix protein associated with induction of proliferation in B lymphocytes. F Biol Chem 1987;262:1 1389-97.

11 Patterson SD, Grossman JS, D'Andrea P, Latter GI. Reduced numatrin/B23/ nucleophosmin labelling in apoptotic Jurkat numatrin/B23/ nucleophosmin labelling in apop

12 Lischwe MA, Smetana K, Olson MO, Busch H. Proteins $\mathrm{C} 23$ and B23 are the major nucleolar silver staining proteins. Life Sci 1979;25:701-8.

13 Buys CHCM, Osinga J. Selective staining of the same set of nucleolar phosphoproteins by silver and Giemsa. A combined biochemical and cytochemical study on staining of NORs. Chromosoma 1984;89:387-96.

14 Miller DA, Breg WR, Warburton D, Dev VG, Miller OJ. Regulation of rRNA gene expression in a human familial 14p+ marker chromosome. Hum Genet 1978;43:289-97.

15 Bauer KD, Bagwell CB, Giaretti W, Melamęd M, Zarbo RJ, Witzig TE, et al. Consensus review of the clinical utility of DNA flow cytometry in colorectal cancer. Cytometry 1993; 14:486-91.

16 Edmin A, Stenling R, Roos G. Prognostic value of DNA content in colorectal carcinoma. A flow cytometric study with some methodologic aspects. Cancer 1987;60:1282-7.

17 Bauer KD, Lincoln ST, Vera-Roman JM, Wallemark CB, Chmiel JS, Madurski ML et al. Prognostic implications of proliferative activity and DNA aneuploidy in colonic proliferative activity and DNA aneuploidy
adenocarcinomas. Lab Invest 1987;57:329-35.

18 Gerdes J, Schwab U, Lemke H, Stein H. Production of a mouse monoclonal antibody reactive with a human nuclear mouse monoclonal antibody reactive with a human nuclear antigen associa

19 Cattoretti G, Becker MH, Key G, Duchrow M, Schluter C, Galle J, et al. Monoclonal antibodies against recombinant parts of the Ki-67 antigen (MIB 1 and MIB 3) detect proliferating cells in microwave-processed formalin-fixed paraffin sections. $\mathcal{F}$ Pathol 1992;168:357-63.

20 Dukes CE. The classification of cancer of the rectum. $f$ Pathol Bacteriol 1932;35:323-32.

21 Laemmli UK. Cleavage of structural proteins during the assembly of the head of bacteriophage T4. Nature 1970;227:680-5. 
22 De Angelis P, Stokke T, Smedshammer L, Lothe RA, Meling GI, Rofstad M, et al. p53 expression is associated with a high degree of tumour DNA aneuploidy and incidence of p53 gene mutation, and is localized to the aneuploid component in colorectal carcinomas. Int $\mathcal{F}$ Oncol 1993;3:305-12

23 Lomo J, Smeland EB, Krajewski S, Reed JC, Blomhoff HK. Expression of the $\mathrm{Bcl}-2$ homologue $\mathrm{Mcl}-1$ correlates with survival of peripheral blood B lymphocytes. Cancer Res 1996;56:40-3.

24 Vindeløv LL, Christensen IJ, Nissen NI. A detergenttrypsin method for the preparation of nuclei for flow cytometric DNA analysis. Cytometry 1983;3:323-7.

25 Shankey TV, Rabinovitch PS, Bagwell B, Bauer KD, Duque RE, Hedley DW, et al. Guidelines for implementation of clinical DNA cytometry. International Society for Analytical Cytology. Cytometry 1993;14:472-7.

26 MacCarty WC, Haumeder E. Has the cancer cell any differential characteristics? Am $\mathcal{f}$ Cancer 1934;20:403-7.

27 Caspersson T.O. In: Cell growth and cell function. A cytochemical study. New York: Norton, 1950.

28 Derenzini M, Betts CM, Ceccarelli C, Eusebi V. Ultrastructural organization of nucleoli in benign naevi and malignant melanomas. Virchows Arch B Cell Pathol 1986;5: maligna
29 Crocker J, Egan MJ. Correlation between NOR sizes and numbers in non-Hodgkin's lymphomas. F Pathol 1988;156 233-9.

30 Yang P, Huang GS, Zhu XS. Role of nucleolar organiser regions in differentiating malignant from benign tumours of the colon. 7 Clin Pathol 1990;43:235-8.

31 Kram N, Nessim S, Geller SA. A study of colonic adenocarcinoma, with comparison of histopathology, DNA flow cytometric data, and number of nucleolar organizer regions. Mod Pathol 1989;2:468-72.

32 Rosa J, Mehta A, Filipe MI. Nucleolar organizer regions, proliferative activity, and DNA index in gastric carcinoma. Histopathology 1990;16:614-6.

33 Giri DD, Nottingham JF, Lawry J, Dundas SAC, Underwood JCE. Silver-binding nucleolar organizer regions (AgNORs) in benign and malignant breast lesions: correla tions with ploidy and growth phase by DNA flow cytometry. F Pathol 1989;157:307-13.

34 Hasegawa S, Okamura K, Shimoji T, Miyake K, Yamakawa Y, Sakamoto H. Evaluation of testicular germ cell tumour proliferation using DNA flow cytometry and nucleolar organizer silver staining. Cytometry 1994;15:59-63. 